Methods The HARP study enrolled HIV-positive women aged 25-50 in Burkina Faso (BF) and South Africa (SA). A stratified sampling strategy was used, with $2 / 3$ of women on ART. Three cervical cancer screening methods were evaluated; (1) visual inspection (VIA/VILI); (2) high-risk HPV DNA (HC-2); (3) conventional cytology. Four-quadrant cervical biopsies were obtained among women with abnormalities detected by at least one test or by colposcopy. Results 1252 women were enrolled (628 in BF; 624 in SA). The distribution of CD4 count (cells/ $\mu \mathrm{L}$ ) was similar in both sites: $68 \%$ with CD4 $+\geq 350$ and $10 \%$ with CD4 $<200$. Prevalence of high risk (HR)-HPV was $43 \%$ in BF and $61 \%$ in SA, and decreased with increasing CD4+ count (P-trend $<0.001)$. VIA/VILI positivity was $24 \%$ in $\mathrm{BF}$ and $41 \%$ in $\mathrm{SA}(\mathrm{P}<0.001)$ but did not vary by $\mathrm{CD} 4$ count (P-trend $=0.30)$. Prevalence of abnormal cytology $(\geq$ LSIL \& $\geq$ HSIL) was higher in SA (89\% \& 30\%) than in BF (24\% \& 5\%). 62\% and $97 \%$ women were biopsied in BF and SA respectively, with CIN2+ prevalence of $6 \%$ and 29\%, respectively (155 of 949 evaluated women thus far). CIN2+ prevalence decreased with increasing CD4+ (Table). Sensitivity of the single screening methods to detect CIN-2 decreased with increasing CD4+ count, whilst specificity tended to increase with increased CD4+ count (Table). Overall, HR-HPV DNA was the most sensitive test (94\%) and HSIL+ cytology the most specific (90\%). Further analyses with combination of tests did not show much improvement on performance.

Conclusions Cervical cancer screening tests among HIV-positive women are most sensitive among women with CD4+ count below 200 cells $/ \mu \mathrm{L}$. Screening strategies may vary according to CD4+ count but this will need to be evaluated prospectively.

\section{P5.010 PREVALENCE AND PREDICTORS OF A POSITIVE CERVICAL CANCER SCREENING TEST IN A SEXUALLY TRANSMITTED INFECTION CLINIC IN LILONGWE, MALAWI}

doi:10.1136/sextrans-2013-051184.1057

\begin{abstract}
2N E Rosenberg, ' $\mathrm{G}$ Kamanga, ${ }^{1,2} \mathrm{H}$ Hoffman, 'B Ndalama, ' $\mathrm{C}$ Mapanje, ${ }^{2} \mathrm{~K}$ Powers ${ }^{3,4} \mathrm{G}$ Chiudzu, ${ }^{1,2} \mathrm{~J} \mathrm{Tang},{ }^{1,2} \mathrm{~S}$ Gopal, ${ }^{1,2} \mathrm{M}$ Hosseinipour. 'UNC Project, Lilongwe, Malawi; ${ }^{2}$ University of North Carolina, Chapel Hill, NC, United States; ${ }^{3}$ Kamuzu Central Hospital, Lilongwe, Malawi; ${ }^{4}$ Ministry of Health, Lilongwe, Malawi
\end{abstract}

Background In Malawi, cervical cancer is the most common cancer among females and a leading cause of cancer-related mortality. Cervical cancer can be averted if pre-cancerous lesions are detected early and treated. Visual Inspection with Acetic Acid (VIA) is an effective screening method for preventing cervical cancer and is sustainable in resource-limited settings. We assessed prevalence and predictors of a positive VIA following its introduction in a sexually transmitted infection (STI) clinic in Lilongwe, Malawi.

Methods From October 2012 to January 2013 all females 25-45 years and females $<25$ years at clinician discretion received VIA screening at the Kamuzu Central Hospital STI Clinic. We calculated the prevalence of a positive VIA result and used logistic regression to identify predictors of a positive result.

Results During this 3.5-month period, 86 women had VIA screening results. Median age was 29,77\% were married, $43 \%$ had at least some secondary education. Forty three percent were HIV-infected and $63 \%$ had an STI using Malawi's syndromic management algorithm. Nineteen percent were VIA-positive, 79\% VIA-negative, and $2 \%$ VIA-uncertain. The prevalence of a VIA-positive result was $7 \%$ in HIV-uninfected women and $33 \%$ in HIV-infected women. Factors significantly associated with a positive VIA result were HIV infection (OR: 6.1, 95\% CI: 1.5, 24.4) and pain during intercourse (OR: 4.5, 95\% CI: $1.2,16.1$ ). Genital warts (OR: $2.4,95 \%$ CI: 0.5 , 10.8) and genital ulcers (OR: 3.1, 95\% CI: 0.5, 20.3) were associated with an increased odds of being VIA-positive, though this trend was not statistically significant.
Conclusions The prevalence of an abnormal VIA was high among Malawian women attending an STI clinic, especially for those with HIV. To prevent cervical cancer mortality, further expansion of VIA screening is needed in Malawi for women at high risk.

\section{P5.011 ARE AUSTRALIAN GENERAL PRACTITIONERS (GPS) AND PRACTISE NURSES (PNS) EQUIPPED FOR INCREASED CHLAMYDIA TESTING? FINDINGS FROM THE AUSTRALIAN CHLAMYDIA CONTROL EFFECTIVENESS PILOT (ACCEPT)}

doi:10.1136/sextrans-2013-051184.1058

${ }^{1}$ R Lorch, ${ }^{2} \mathrm{~A}$ Yeung, ${ }^{2} \mathrm{~J} S$ Hocking, ${ }^{2} \mathrm{~A}$ Vaisey, ${ }^{2,3} \mathrm{C}$ K Fairley, ${ }^{1} \mathrm{~B}$ Donovan, ${ }^{1} \mathrm{M}$ Law, ${ }^{4} \mathrm{M}$ Temple-Smith, 'R Guy. 'Kirby Institute, University of New South UK, Coogee, Sydney, Australia; ${ }^{2}$ Centre for Women's Health, Gender and Society, Melbourne School of Population Health, University of Melbourne, Melbourne, Australia; ${ }^{3}$ Melbourne Sexual Health Centre, Carlton, Melbourne, Australia; ${ }^{4}$ Department of General Practice, University of Melbourne, Melbourne, Australia

Background ACCEPt, a large cluster randomised control trial, aims to determine if annual testing for 16 to 29 year olds in general practise can reduce chlamydia prevalence. ACCEPt is also the first trial investigating the potential role of the $\mathrm{PN}$ in chlamydia testing. To inform the intervention, GPs' and PNs' chlamydia knowledge and practises were explored.

Methods GPs and PNs from 143 clinics were recruited from 54 postcodes in 4 Australian States and asked to complete a survey at time of recruitment. Survey responses were compared using conditional logistic regression to account for GPs and PNs from the same clinics participating.

Results Of 607 GPs and 126 PNs enrolled in ACCEPt, 86\% and $78 \%$ completed the questionnaire, respectively. A third of GPs (32\%) compared to $23 \%$ of $\mathrm{PNs}(\mathrm{P}=0.076)$ correctly identified the two age groups with the highest infection rates in women and only $17 \%$ vs $16 \%(\mathrm{P}=0.942)$ identified the correct age groups in men. Fewer GPs than PNs would offer testing opportunistically to asymptomatic young patients, including women having a Pap smear (55\% vs. $84 \%$, $\mathrm{P}=<0.001)$; antenatal checkup (44\% vs. $83 \%, \mathrm{P}=<0.001)$ and Aboriginal men with a sore throat ( $33 \%$ vs. $79 \%, \mathrm{P}=<0.001)$. Fewer GPs than PNs knew that retesting was recommended after chlamydia treatment ( $87 \%$ vs. $93 \%, \mathrm{P}=0.027)$; and that the recommended timeframe for retesting was 3 months (26\% vs $66 \%$, $\mathrm{P}=<0.001)$. Under half of $\mathrm{PNs}(41 \%)$ reported involvement in chlamydia testing, with $79 \%$ wanting greater involvement and $87 \%$ wanting further training.

Conclusion Our survey reveals more gaps in chlamydia knowledge and management among GPs than PNs, which may be contributing to low testing rates in general practise. PNs have a role in increasing chlamydia testing.

\section{P5.012 IS THE STAGE OF THE MENSTRUAL CYCLE RELATED TO CHLAMYDIA DETECTION?}

doi:10.1136/sextrans-2013-051184.1059

1.2D S Forcey, ${ }^{1,3} \mathrm{~J}$ S Hocking, ${ }^{1,4}$ S Tabrizi, ${ }^{1,3} \mathrm{C}$ S Bradshaw, 1,3M Y Chen, 1,3G Fehler, 'J L Nash, ${ }^{1,3} \mathrm{C}$ K Fairley. 'Melbourne Sexual Health Centre, Carlton, Australia; ${ }^{2} T$ he University of Melbourne, Melbourne, Australia; ${ }^{3}$ Melbourne School of Population Health, University of Melbourne, Melbourne, Australia; ${ }^{4} T$ he Royal Women's Hospital, Melbourne, Australia

Background We investigated the association between chlamydia detection and stage in the menstrual cycle to investigate whether chlamydia detection was higher at different stages of the cycle.

Methods Electronic medical records for women attending Melbourne Sexual Health Centre March 2011 - 31 $1^{\text {st }}$ December 2012, who were tested for chlamydia by nucleic acid amplification of high vaginal, cervical, or urinary samples, and who recorded a date of Last Normal Menstrual Period (LNMP) between 0-28 days were 